\title{
Morphology transitions in three-dimensional domain growth with Gaussian random fields
}

\author{
Belita Koiller \\ Instituto de Física, Universidade Federal do Rio de Janeiro, Cx.P. 68.528, 21945-970, RJ, Brazil \\ Mark O. Robbins \\ Department of Physics and Astronomy, Johns Hopkins University, Baltimore, MD 21218
}

(March 31, 2000)

We study the morphology of magnetic domain growth in disordered three dimensional magnets. The disordered magnetic material is described within the random-field Ising model with a Gaussian distribution of local fields with width $\Delta$. Growth is driven by a uniform applied magnetic field, whose value is kept equal to the critical value $H_{c}(\Delta)$ for the onset of steady motion. Two growth regimes are clearly identified. For low $\Delta$ the growing domain is compact, with a self-affine external interface. For large $\Delta$ a self-similar percolation-like morphology is obtained. A multi-critical point at $\left(\Delta_{c}, H_{c}\left(\Delta_{c}\right)\right)$ separates the two types of growth. We extract the critical exponents near $\Delta_{c}$ using finite-size scaling of different morphological attributes of the external domain interface. We conjecture that the critical disorder width also corresponds to a maximum in $H_{c}(\Delta)$.

\section{INTRODUCTION}

Many physical processes involve motion of the interface between two domains through a disordered media. Classic examples are magnetic domain growth, fluid invasion of porous media and fluid segregation in gels. 1 . 2 Previous studies of such systems have shown that changing the strength of disorder leads to critical transitions between different growth morphologies 311 Most theoretical work on the subject has been done on models with an underlying crystalline lattice of spins or pores. The crystalline anisotropy may lead to a faceted growth regime in the low disorder limit. As disorder increases, there may be a transition to compact growth with a self-affine interface. In the high disorder limit, growing domains are self-similar fractals, characteristic of percolation.

Studies of driven interfaces in the zero-temperature random-field (and random-bond) Ising model (RFIM) indicate that the dimensionality, coordination number, and distribution of random fields are all important in $\mathrm{ge}_{\mathrm{g}}$ termining the sequence of morphological transitions. 5 In two dimensions (2D), there is a transition from selfsimilar to faceted growth at a critical value of disgrder, if the the distribution of random fields is bounded.5 The critical behavior is not universal, and can be related to the analytic form of the tails in the distributiond For an unbounded Gaussian distribution, percolative growth occurs for any finite strength of disorder. In the three dimensional (3D) RFIM with a bounded distribution of dis- order all three types of growth morphology, faceted, selfaffine, and pefcolative, are found as the strength of disorder increases.6 Analysis of growth probabilities suggests that the transition from percolative to self-affine growth might be universal, but that the self-affine to faceted transition is non-universal and could be suppressed by an unbounded distribution of fields.

The suggestion that faceted growth may be eliminated by unbounded distributions of disorder in 3D is supported by renormalization group studies of equilibrium interface conformations 12 Calculations for a Gaussian distribution of random fields show that faceted interfaces are suppressed by any amount of disorder in dimensions $d \leq 3$. Porous media and some magnetic systems do not have any underlying crystalline structure, and it is only introduced in the models for computational convenience. Thus suppression of faceted growth by unbounded distributions of disorder leads lattice models to provide a more accurate description of real systems.

In this paper we explore the effect of unbounded Gaussian disorder on growth morphology transitions in the 3D RFIM at zero temperature. We find that this unbounded distribution does indeed eliminate the faceted growth regime. There is a single transition from selfaffine growth at low disorder to percolative growth at high disorder. For each value of the disorder we find the critical field $H_{c}$ needed to initiate steady growth. The critical behavior at the onset of growth is analyzed, and the critical exponents are consistent with previous results for bounded distributions of random fields.

The transition between self-similar and self-affine growth is shown to be a multi-critical point. We identify lengths that diverge as the strength of disorder is varied along the line of critical fields, and evaluate critical exponents. Our analysis reveals problems with previous work on bounded distributions of random fields. These studies identified a "fingerwidth" with the cprrelation length that diverges at the multi-critical point. 6 However, our work with larger systems shows that this fingerwidth does not diverge, and its saturation leads to errors in the determination of critical properties. New work will be needed to determine whether the self-affine to percolative transitions for bounded and unbounded distributions are in the same universality class. However, we can compare our results to studies of magnetic hysteresis in the $3 \mathrm{R}$ RFIM with Gaussian random fields by Perković et al.9.10 These 
studies use a different growth algorithm, and we find that this leads to changes in the critical disorder and critical exponents.

The paper is organized as follows. In Sec. II we describe the growth model. Studies of the critical field and critical exponents at the onset of motion are presented in Sec. III A. The transition in growth morphology is examined in Sec. IIIB, and Sec. IV contains our general summary and conclusions.

\section{GROWTH MODEL AND ALGORITHM}

The energy of a system of Ising spins $\left(s_{i}= \pm 1\right)$ at the sites $i$ of a simple cubic lattice is written as

$$
\mathcal{H}=-\sum_{<i, j>} s_{i} s_{j}-\sum_{i}\left(\eta_{i}+H\right) s_{i} .
$$

The first term on the right-hand side of Eq. (11) represents the ferromagnetic coupling between nearest neighbor spins, with the exchange coupling taken as the energy unit. The second term gives the interaction of each spin with the uniform external magnetic field $H$ and with the random local field $\eta_{i}$. The local fields are uncorrelated, following a Gaussian distribution function whose width $\Delta$ quantifies the degree of disorder:

$$
P(\eta)=\left(2 \pi \Delta^{2}\right)^{-1 / 2} e^{-\eta^{2} /\left(2 \Delta^{2}\right)} .
$$

As in previous studies, 8 the simulation cell is a cube of side $L$, with the lattice constant taken as the unit of length. Periodic boundary conditions are imposed along the $x$ and $y$ directions. All spins are initially anti-parallel to the external field, i.e. $s_{i}=-1$, except those in the bottom layer, $z=1$. This layer constitutes the "seed" for growth of the +1 domain, which is driven by the external field. The orientation of this seed plane does not affect critical behavior in the self-affine and self-similar growth regimes of interest here, 13.14 but does affect the faceted greyth seen for weak, bounded distributions of disorder. 15.16

Growth proceeds through single spin flips at zero temperature. Whenever a spin flip from $s_{i}=-1$ to $s_{i}=+1$ lowers the total energy, it is implemented. However, only spins on the growing interface are allowed to flip. This restriction is motivated by the fluid invasion problem, 50 and differentiates our dynamics from the model considered by Perković et al. 10 in studies of hysteresis in magnetic systems.

We have developed a new memory-efficient algorithm that allows us to consider cells with $L$ as large as 1152 in the present study. The simulation cell is subdivided into smaller cubic cells of side $s$. The $s^{3}$ spins of a sub-cell remain active in memory only while at least one of them is a "flippable" interface spin. Growth occurs at fixed driving field $H$. This allows the local random field to be encoded into a single byte that gives the minimum number of additional neighbors needed to flip the spin at that site. This number is decreased by one every time the spin acquires a new +1 neighbor. When enough neighbors are present, the spin is flipped. For reasons discussed in Sec. IIIB, we flipped all spins that were completely surrounded by +1 neighbors, regardless of their local field. Such spins can not affect growth at any other site, and being able to remove the cells containing them from memory allowed us to treat larger systems. Preliminary runs where these spins were not flipped gave equivalent results.

At each $L$ and $\Delta$, domains were grown in an ensemble of samples with different configurations of $\left\{\eta_{i}\right\}$. In each sample, growth was stopped when all spins on the interface were stable, or when the interface first reached the top of the system $(z=L)$. The morphology of the domain was then analyzed as described below.

\section{RESULTS}

\section{A. The critical field}

The external field $H$ provides a driving force that causes the domain of +1 spins to grow. If $H$ is too small, the domain wall will remain pinned near the bottom of the system, while large $H$ will cause all spins to flip. Figure 1 shows the probability, $P_{t o p}$, for a domain to grow to the top of the cell as a function of $H$ at several different system sizes. Results for two values of $\Delta$ corresponding to self-affine $(\Delta=2.1)$ and self-similar $(\Delta=3.6)$ growth are shown. For each $\Delta$, the range of $H$ over which the probability rises from 0 to 1 becomes narrower as $L$ increases. All of the curves intersect at a critical field $H_{c}(\Delta)$ and probability $P_{c}(\Delta)$. If $H>H_{c}$, the probability approaches unity in the thermodynamic limit $(L \rightarrow \infty)$, while the probability vanishes in this limit if $H<H_{c}$.

Previusties with a uniform distribution of random fields 3 8 17,18 have shown that there is a diverging correlation length $\xi_{H} \sim\left|H-H_{c}\right|^{-\nu_{H}}$ as $\left|H-H_{c}\right| \rightarrow 0$. The critical exponent in the self-similar growthregime $\theta$ is consistent with the 3D percolation exponent $19 \nu_{H}=8,8 \pm$ 0.02 , while $\nu_{H}=0.75 \pm 0.02$ for self-affine growth. 6.18

Near $H_{c}$, the probability for the domain to span the system should only depend on the ratio of system size to $\xi_{H}$. This suggests that when probability is plotted against $\left(H-H_{c}\right) L^{1 / \nu_{H}}$ the results for all system sizes should collapse onto a universal curve. Fig. 2 verifies that the data from Fig. 11 are consistent with this ansatz, and with the values of $\nu_{H}$ that were found for uniform distributions of random fields. 

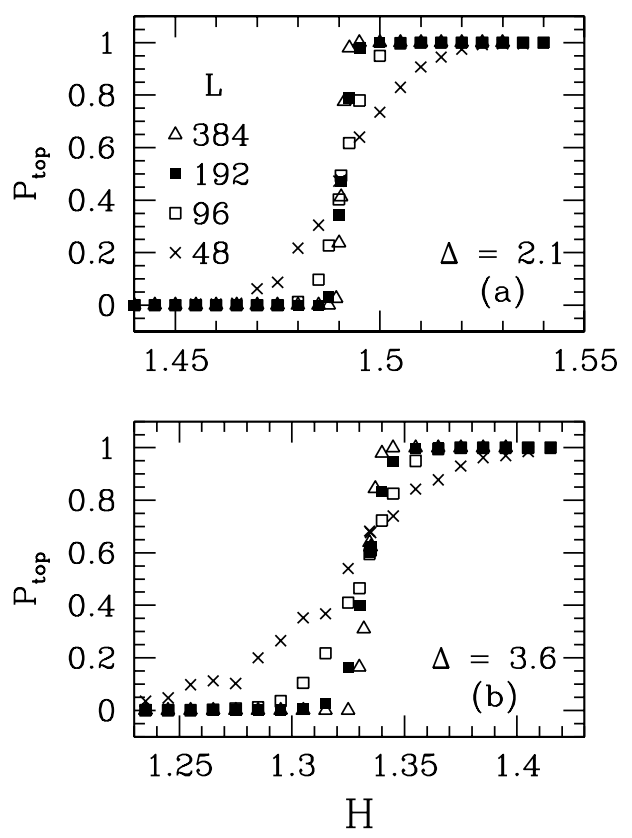

FIG. 1. Probability $P_{t o p}$ that the domain grows to the top of the system as a function of $H$ at the indicated system sizes $L$ for (a) $\Delta=2.1$ (self-affine) and (b) $\Delta=3.6$ (self-similar). Four hundred different systems were used for each $L$ and $\Delta$, so the statistical uncertainty is less than \pm 0.025 (symbol size).

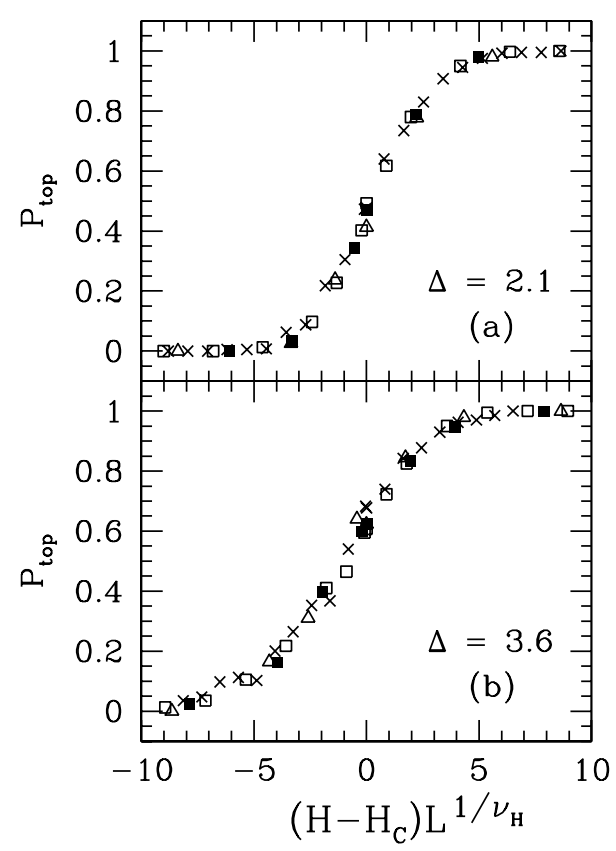

FIG. 2. Probability that the domain grows to the top of the system as a function of $\left(H-H_{c}\right) L^{1 / \nu_{H}}$ for (a) $\Delta=2.1$ and (b) $\Delta=3.6$. In (a) $H_{c}=1.4905$ and $\nu_{H}=0.75$. For (b) $H_{c}=1.335$ and $\nu_{H}=0.88$. The symbols for each $L$ are the same as in Fig. 11. Four hundred different systems were used for each $L$ and $\Delta$, so the statistical uncertainty is less than \pm 0.025 (symbol size).
Examination of the spanning probability for other values of $\Delta$ shows that $P_{c}(\Delta)$ is always near $2 / 3$ for selfsimilar orowth and decreases with $\Delta$ in the self-affine regime.20 To determine $H_{c}(\Delta)$ we worked with the largest accessible system size ( $L=768$ or 1152$)$ and found the value of $H$ that gave a spanning probability between 0.5 and 0.7. This determines $H_{c}$ with an accuracy of about 0.0001 . We found that even larger errors in the growth field did not change the morphology of spanning domains that is analyzed in the next section.

In Figure 3 we show the variation of $H_{c}$ with disorder for the range of interest in the present study. At small $\Delta$ the value of $H_{c}$ increases monotonically. There is a maximum near $\Delta=2.5$, and then $H_{c}$ drops monotonically, becoming negative for $\Delta \gtrsim 7$.

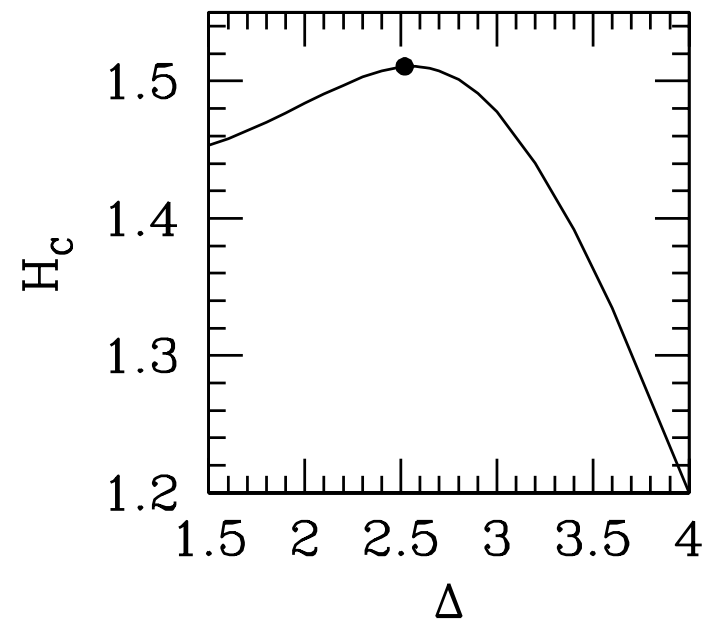

FIG. 3. Variation of the critical field $H_{c}$ with Gaussian disorder $\Delta$. A filled circle indicates the value of $\Delta_{c}=2.52 \pm 0.03$ determined below. Self-affine growth occurs for $\Delta<\Delta_{c}$ and self-similar growth occurs at larger $\Delta$.

\section{B. Transition in Growth Morphology}

As expected, the faceted growth regime seen for bounded distributions of random fields 6 was suppressed by Gaussian disorder. The only transition that we observed was from self-similar growth at high disorder to self-affine growth at low disorder.

Self-similar growth is isotropic, while self-affine growth has a well-defined direction at long-length scales. Previous work on other models 3 shows that the transition between these two growth regimes is a multi-critical point at some $H_{c}$ and $\Delta_{c}$. An order parameter can be defined in analogy to equilibrium magnetic transitions as the average of the unit vector normal to the interface. 13 For $\Delta<\Delta_{c}$ this average is finite, while for $\Delta>\Delta_{c}$ the order parameter vanishes. A correlation length, $\xi$, that diverges at $\Delta_{c}$ can also be defined. As $\Delta$ approaches $\Delta_{c}$ from above (self-similar regime), longer and longer segments of the domain wall advance in the same direction. In the self-affine regime, deviations from the mean di- 
rection occur over longer and longer length scales as $\Delta$ increases to $\Delta_{c}$. In the following subsections we examine the morphology of domains and use finite-size scaling to determine $\Delta_{c}$ and the exponent $\nu$ that describes the divergence of $\xi$ as $\Delta \rightarrow \Delta_{c}$.

\section{Fingerwidths}

Previous experimental 11 and theoretical 3 studies have used a simple measure of the range of correlations. A fingerwidth $w$ was calculated by examining lines of adjacent nearest-neighbor spins, and averaging the length of contigues segments of +1 spins (or fluid-invaded

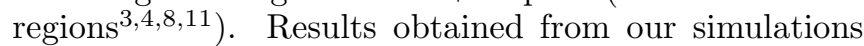
are given in Figure $4(\mathrm{a})$. As in previous work, $w$ is independent of $L$ at high disorder and proportional to $L$ at low disorder. If one assumes that $w$ diverges at $\Delta_{c}$ in the thermodynamic limit, $w \propto\left(\Delta-\Delta_{c}\right)^{-\nu}$, then one can determine $\Delta_{c}$ and $\nu$ from finite-size scaling collapses of the fingerwidth data. While this assumption was used in previous studies, our results with larger system sizes indicate that it is not justified. Finite-size scaling collapses become worse and worse as the range of $L$ increases, and give estimates for $\Delta_{c}$ that are clearly in the self-affine regime.

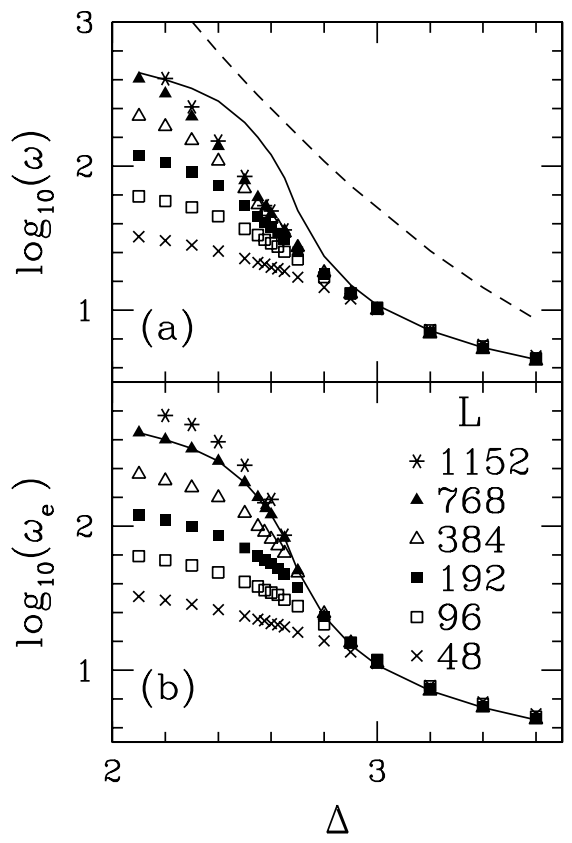

FIG. 4. (a) Fingerwidths $w$ calculated as a function of disorder $\Delta$ for the simulation cell sizes indicated in (b). The dashed line gives an upper bound, obtained from the statistical probability of singlets or pairs of unflippable $s=-1$ spins. The solid line shows the external fingerwidth $w_{e}$ for $L=768$. Panel (b) shows $w_{e}$ vs. $\Delta$ for all $L$. In both panels fingerwidths were calculated in the plane normal to the nominal growth direction, $z$.
The reason that $w$ does not diverge at $\Delta_{c}$ is quite simple. Even in the self-affine regime there are small clusters of unflippable spins (Fig. 5) due to the tails in the distribution of $\eta_{i}$. These clusters are left behind by the advancing interface, and do not affect the morphology at long-length scales. However they do lead to a finite value of $w$. The limiting value of $w$ can be estimated from the probability for single isolated spins, pairs of spins, etc.. The upper bound for $w$ from single spins and pairs is indicated by a dashed line in Fig. 4(a). This upper bound clearly inhibits divergence of the fingerwidth with $L$ at any finite $\Delta$. It is only about 300 at the value of $\Delta_{c} \approx 2.5$ determined below, and lower bounds would be obtained by considering larger clusters.

We attempted to define improved fingerwidths by eliminating all isolated spins, and then all pairs of isolated spins before determining the fingerwidth. However, this procedure was inefficient and did not converge rapidly. In the following we focus entirely on the morphology of the external interface, and thus eliminate surrounded unflipped regions of all sizes. The scaling behavior in the self-affine regime is only associated with the external interface: Once the surrounded regions are left behind, they become irrelevant. In the self-similar regime we know that the external interface of a percolation cluster has the same fractal dimension as the entire cluster. Thus the external interface should give nearly the same fingerwidth as the cluster.

The solid line in Figure 4(a) shows the fingerwidth calculated from the external interface, $w_{e}$, as a function of $\Delta$ at $L=768$. As expected, the unflipped regions have no effect on fingerwidth for $\Delta \gtrsim 3$, where the interface forms a fractal percolation pattern with narrow fingers. However as $\Delta$ decreases towards $\Delta_{c} \approx 2.5$, the solid line rises sharply above the other data points. Figure 1 (b) shows how the external fingerwidth changes with $L$. Finite-size scaling collapses of $w_{e}$ are discussed below.

\section{Interface roughness}

Self-affine interfaces are characterized by the scaling properties of the interface roughness. 13 Due to our periodic boundary conditions, the average direction of the external interface is normal to $z$. Its position is given by a height $h(x, y)$ that may be multivalued. The roughness $\rho(\ell)$ over a square region of side $\ell$ in the $x-y$ plane can be quantified by the root-mean-squared ( $\mathrm{rms}$ ) variation in $h$

$$
\rho(\ell)=\sqrt{\left\langle(h-\langle h\rangle)^{2}\right\rangle_{\ell}},
$$

where $\langle h\rangle$ is the average over a given square and \langle\rangle$_{\ell}$ indicates an average over all square regions of side $\ell$. For a self-affine interface, 1 , $\rho(\ell) \sim \ell^{\alpha}$ at large $\ell$. The roughness exponent $\alpha<1$ characterizes the degree of anisotropy, and would be unity for a self-similar fractal. 


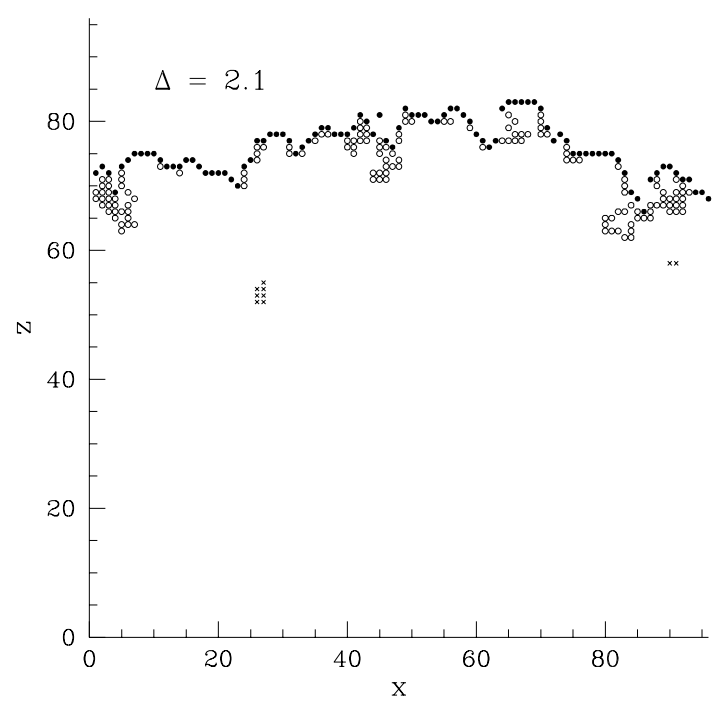

FIG. 5. Cross-section through a system of size $L=96$ for $\Delta=2.1$. The circles (open and solid) show all spins on the external interface. Solid circles indicate the single-valued interface obtained by taking the highest spin at each point in the $(\mathrm{x}, \mathrm{y})$ plane. Overhangs are evident in the regions where the solid and open circles separate. These form as the interface grows around unflippable clusters (crosses). As discussed in the text, unflippable clusters prevent the total fingerwidth $w$ from diverging. However, the interface in this figure is clearly self-affine, and the largest unflippable cluster is much smaller than the system size.

Figure 5 shows a cross-section through a system of size $L=96$ for $\Delta=2.1$, which is well into the self-affine regime. The external interface (circles) is a multi-valued function. In some regions there are overhangs where the interface extends over itself. These overhangs are necessary if the interface is to grow around small clusters of unflippable spins (crosses). The size of overhangs and of clusters of unflipped spins increases as $\Delta$ rises to $\Delta_{c}$.

Previous work 17 shows that overhangs can change the scaling of $\rho(\ell)$ at small $\ell$. One way of highlighting their effect is to compare $\rho(\ell)$ with the roughness $\rho_{t}(\ell)$ of the single-valued interface, $h_{t}(x, y)$, obtained by taking the top (highest) point on the external interface at each $\ell$ (closed circles in Fig. 5). Figure 6 shows log-log plots of both quantities vs. $\ell$ at the indicated values of $\Delta$. For $\Delta \leq 2.2$, results for the external (solid symbols) and single-valued (open symbols) interfaces converge at large $\ell$. The slope of the curves in the converged region is consistent with the roughness exponent $\alpha=2 / 3$ that is predicted from scaling arguments 2123 and observed in previous simulations. 18 At small $\ell$ the dashed and full lines separate due to overhangs. The value of $\rho_{t}$ goes to zero at $\ell=1$, while the value of $\rho$ goes to the rms variation in height above a single point in the $(x, y)$ plane. The growing separation between dashed and full lines as $\Delta$ increases towards $\Delta_{c}=2.52$ shows that the size of the overhangs increases. For $\Delta=2.5$ there is no convergence of the lines even at the largest $\ell$ and $L$ we could study. The overhang size is one measure of a diverging length as $\Delta$ approaches $\Delta_{c}$ from below, and is analyzed in following sections.

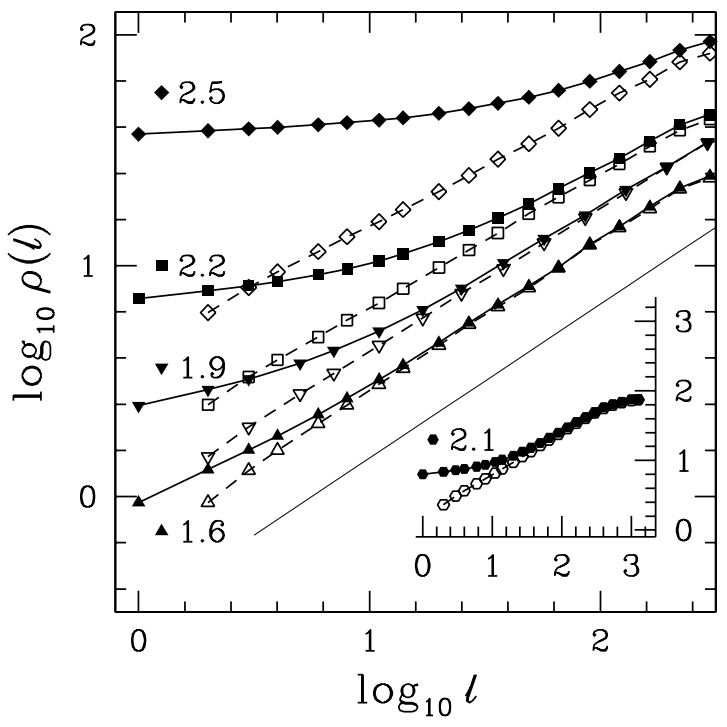

FIG. 6. External interface roughness at the indicated values of disorder $\Delta$ for $L=768$. The full lines and symbols correspond to the total interface $\rho$, while the dashed lines and open symbols refer to the single-valued top interface $\rho_{t}$. The straight solid line below the curves shows the slope corresponding to $\alpha=\frac{2}{3}$. The inset at the lower right shows that $\rho$ and $\rho_{t}$ converge for $\Delta=2.1$ when a larger system size is used $(L=1260)$. There is some rounding of the curves at large $\ell / L$ due to finite-size effects.

\section{Determining $\Delta_{c}$}

The data in Figures 14 and 6 give clear evidence of a morphological transition, and rough bounds on the value of $\Delta_{c}$. The saturation of the external fingerwidth with increasing $L$ for $\Delta \gtrsim 2.65$ gives an upper bound for $\Delta_{c}$ (Fig. (1)(b)), while the merging of $\rho$ and $\rho_{t}$ at large $\ell$ for $\Delta \leq 2.2$ gives a lower bound (Fig. 6). In this subsection we investigate other morphological attributes of the external interface that provide more accurate bounds for $\Delta_{c}$. In the next subsection we determine the exponent $\nu$ through finite size scaling analysis of these quantities.

A single-valued interface, $h_{b}(x, y)$, can also be defined by taking the bottom (lowest) value of the external interface $h$ for each $(x, y)$. Figure $\mathbb{E}(\mathrm{a})$ shows the average heights of the top and bottom interfaces, $\left\langle h_{t}\right\rangle$ and $\left\langle h_{b}\right\rangle$, as a function of $\Delta$ at various system sizes. In the self-similar regime, the fractal external interface extends throughout the entire height of the cell. The value of $\left\langle h_{t}\right\rangle$ is a constant fraction of the system size and $\left\langle h_{b}\right\rangle$ is of order of the fingerwidth. The two averages converge in the self-affine regime, where the difference between them, $d h \equiv\left\langle h_{t}\right\rangle-\left\langle h_{b}\right\rangle$, is a measure of the height and abundance of overhangs. 


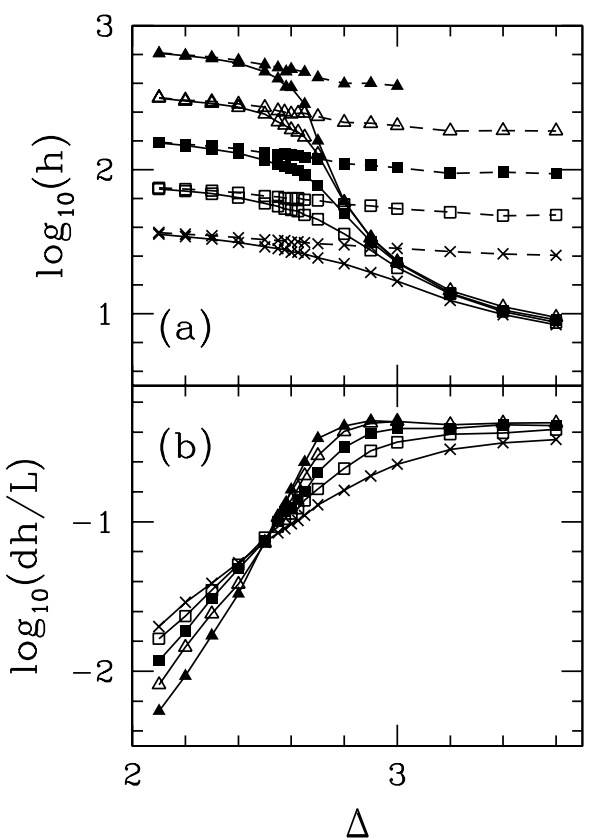

FIG. 7. (a) Average heights of the single-valued interfaces $\left\langle h_{t}\right\rangle$ (dashed lines) and $\left\langle h_{b}\right\rangle$ (solid lines) as a function of $\Delta$ for system sizes ranging from $L=48$ (crosses) to $L=768$ (solid triangles), following the symbol definitions in Figure 4. (b) Average height difference $d h \equiv\left\langle h_{t}-h_{b}\right\rangle$, normalized by system size, as a function of disorder. The crossing point where curves for all $L$ intersect gives an estimate for $\Delta_{c}$.

In Figure 7(b) we plot $d h / L$ vs. $\Delta$. As implied by the above discussion, this ratio vanishes at small $\Delta$ and rises to a constant fraction at large $\Delta$. The increase becomes sharper with increasing $L$, and there is a clear crossing of all curves at $\Delta \approx 2.5$. This means that for $\Delta$ below the crossing point $d h / L$ decreases with $L$, while above the crossing point $d h / L$ increases with system size. We conclude that the crossing point must coincide with $\Delta_{c}$.

We have examined a variety of other quantities to confirm that all give consistent values of $\Delta_{c}$ and to minimize the error bars. Figure 8 shows results for two probabilities that are related to the global minimum, $h_{-}$, of each external interface. Data points connected by solid lines give the probability that $h_{-} / L$ is greater than $1 / 3$. This probability is unity in the self-affine limit and drops to zero in the self-similar regime where the fractal external interface extends all the way to the bottom of the system. The data points connected by dashed lines in Figure 8 give the probability that $h_{-}$remains at the height of the initial seed plane. This probability is unity in the selfsimilar regime and drops to zero in the self-affine regime. Both probabilities exhibit sharper transitions from one to zero as $L$ increases, and should become step-functions at $\Delta_{c}$ in the limit $L \rightarrow \infty$. Crossing points for the two probabilities in Fig. 8, the interface width in Fig. 7(b), and all other quantities that we examined are consistent with $\Delta_{c}=2.52 \pm 0.03$.

It is interesting to note that Fig. 3 shows a maxi- mum in $H_{c}(\Delta)$ at $\Delta_{c}$. This is a reasonable result, given the difference in growth mechanisms for self-affine and self-similar regimes. In the self-affine regime, the interface must advance across the entire width of the system. Thus $H_{c}$ is sensitive to the regions that are hardest to flip, and rises with $\Delta$. In the self-similar regime, the interface follows the path of least resistance. Since the number of spins that must be flipped (the percolation probability) is less than $1 / 2$, increasing $\Delta$ makes it easier to flip enough spins to span the system, and decreases $H_{c}$. The rate of decrease in $H_{c}$ can be calculated exactly from the percolation probahility in the large $\Delta$ limit, where spins are decorrelated 6 . $d H_{c} / d \Delta=-0.4907$.

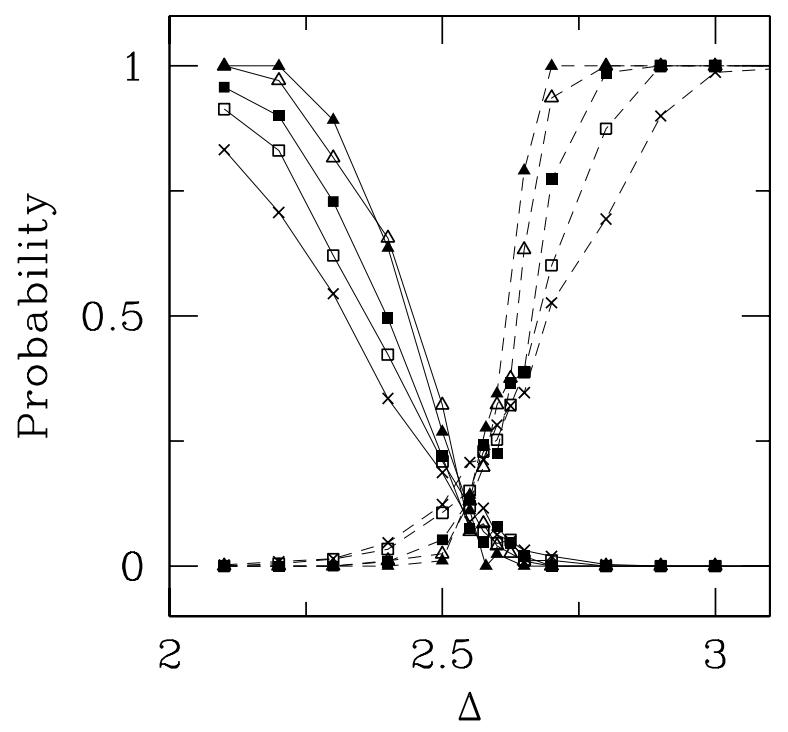

FIG. 8. Probabilities related to the global minimum $h_{-}$of the full external interface. The data points connected by solid lines give the probabilities that $h_{-} \geq L / 3$, while the data points connected by dashed lines give the probability that at least one point on the external interface remains at the initial seed plane. System sizes range from $L=48$ (crosses) to $L=768$ (solid triangles), following the symbol definitions in Figure As As $L$ increases, the probabilities show a sharper change from zero to one. Quantities were averaged over an ensemble of at least 100 samples at each value of $\Delta$ and $L$.

\section{Finite-size scaling determination of $\nu$}

As in Sec. III A, we use finite-size scaling to determine the exponent $\nu$ that describes the diverging correlation length $\xi$ at $\Delta_{c}$. The deviation from $\Delta_{c}$ is measured by $\delta \equiv\left(\Delta-\Delta_{c}\right) / \Delta$. We assume that $\xi \sim \delta^{-\nu}$, and that close to the critical disorder the only relevant lengths are $\xi$ and the system size $L$. Then dimensionless quantities like those shown in Figs. 7 (b) and 8 can only depend on $L / \xi$, or equivalently $L^{\mathrm{H} \nu} \delta$. When plotted against $L^{1 / \nu} \delta$, results for all system sizes should collapse onto a 
universal scaling function.

Figure 9 shows a scaling collapse for the data of Fig. 7(b). The scaled interface widths $d h / L$ for system sizes $L=48,96,192,384$ and 768 collapse well onto a universal curve near $L^{1 / \nu} \delta=0$. As $\left|L^{1 / \nu} \delta\right|$ increases, the curves for small $L$ begin to deviate from the others. These deviations reflect corrections to scaling. They appear first at small $L$ because these data points are for larger values of $\delta$ than their counterparts at large $L$.

Since the magnitude of corrections to scaling is not known, there is some uncertainty in determining the values of $\Delta_{c}$ and $\nu$. We found acceptable collapses for $d h / L$ with $\Delta_{c}=2.52 \pm 0.03$ and $\nu=2.5 \pm 0.3$. Scaling collapses of other quantities related to the interface width, including $\rho(1)$, the ratio of the lowest and highest points on the entire interface, and the probabilities shown in Figure 8, all gave consistent ranges of $\Delta_{c}$ and $\nu$. We also considered the scaling variable $\delta^{\prime} \overline{\overline{0}}\left(\Delta-\Delta_{c}\right) / \Delta_{c}$, which gives different, and often larger 10.17 corrections to scaling. This led to a narrower region of scaling, but the same range of values for $\nu$ and $\Delta_{c}$.

The scaling behavior of the external fingerwidth is more complicated, because the distribution of fingerwidths becomes bimodal in the self-affine regime. Most of the fingerwidths are essentially equal to the system size $L$. However, there is a significant fraction of very small fingerwidths from the region of width $d h$ near the top of the interface (see Fig. 5). These make a disproportionate contribution to $w_{e}$ that does not obey the scaling ansatz.

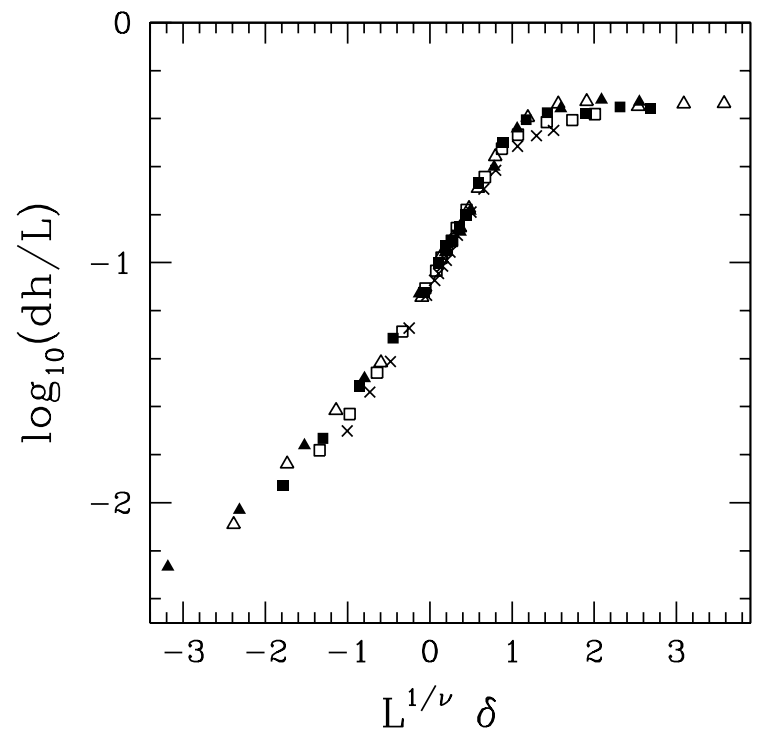

FIG. 9. Finite size scaling collapse of the data in Figure (b) for $\Delta_{c}=2.52$ and $\nu=2.4$. The symbols used for each $L$ are the same as in Figure 4 .

The contribution of small fingerwidths decreases if one calculates higher moments of the fingerwidth. We define

$$
w_{e n}=\sqrt[n]{\left\langle w_{e}^{n}\right\rangle}
$$

where $n=1$ gives the mean width, $n=2$ gives the rms width, etc.. We find a steady improvement in finite-size scaling collapses with increasing $n$. Figure 10 shows that results for $w_{e 4} / L$ collapse onto a universal curve at large $L$ with the same $\Delta_{c}$ and $\nu$ used in Fig. 9. Best fits for $\nu$ increased consistently from $2.0 \pm 0.2$ at $n=1$ to $2.2 \pm 0.2$ for $n=4$, and the quality of the collapse showed progressive improvement.

If $w_{e}$ is proportional to the diverging correlation length, then it must diverge with the same exponent. To check this, we examined the slope of plots of $\log _{10} w_{e n}$ against $\log _{10} \delta$. The slopes were indeed consistent with values of $\nu$ from finite-size scaling, although the uncertainties were somewhat larger.

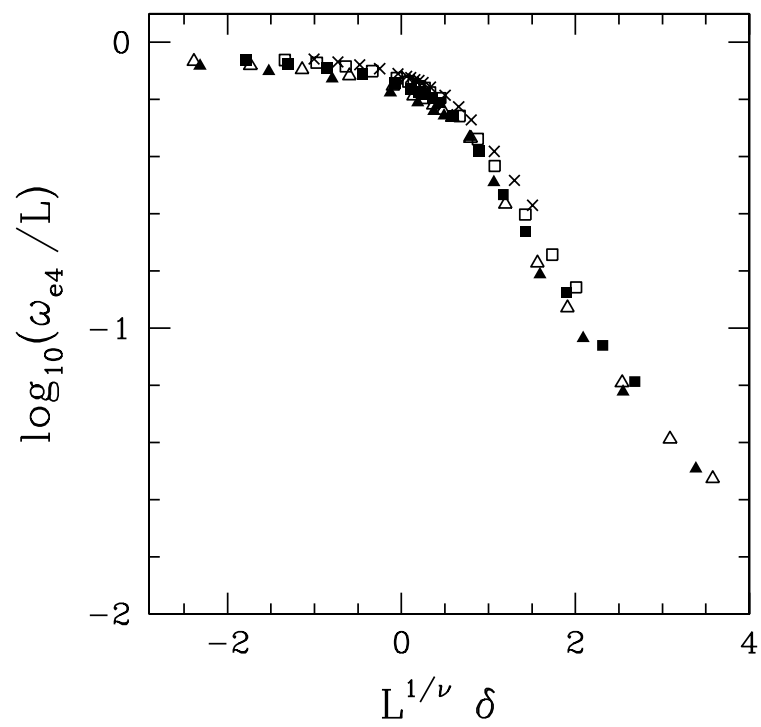

FIG. 10. Finite size scaling collapse of $w_{e 4}$ using $\Delta_{c}=2.52$ and $\nu=2.4$. The symbols used for each $L$ are the same as in Figure 4 .

\section{SUMMARY AND DISCUSSIONS}

We have studied the zero-temperature phase diagram for interface growth in the 3D RFIM with a Gaussian distribution of random fields. There is a single transition from self-affine growth below $\Delta_{c}$ to percolative growth at larger disorder. The tails of the Gaussian distribution eliminate the faceted regime obseryed in previous 3D studies of bounded field distributions. 6 This means that the Gaussian RFIM provides a more realistic description of transitions in systems that do not have an underlying crystalline lattice, such as random porous media or amorphous magnets. The phase diagram is also quite different from that for the 2D RFIM where Gaussian randomness suppresses the self-affine regime.

The critical behavior at the onset of motion in the self-affine and self-similar regimes was analyzed using finite-size scaling. The critical exponent $\nu_{h}$ that describes the diverging length scale as $H \rightarrow H_{c}$ was found to be 
$0.75 \pm 0.02$ for self-affine growth and $0.88 \pm 0.02$ for selfsimilar growth. These values are consistent with results for bounded distributions of disorder 6 We also found the same roughness exponent $\alpha=2 / 3$ in the self-affine regime (see Fig. 6). These results indicate that changing the form of the distribution of random fields does not change the universality class of the self-affine and selfsimilar growth regimes.

The multi-critical point that separates self-affine and percolative growth was also analyzed. We found that the fingerwidth used in previous work does not diverge and can not be used to determine $\Delta_{c}$. Examination of the external interface revealed two lengths that did diverge at $\Delta_{c}$ : The overhang size $d h$ diverges as $\Delta$ increases to $\Delta_{c}$ in the self-affine regime, and the external fingerwidth $w_{e}$ diverges as $\Delta$ decreases to $\Delta_{c}$ in the percolative regime. Finite-size scaling collapses of these and other quantities gave consistent values for $\Delta_{c}=2.52 \pm 0.03$ and $\nu=$ $2.4 \pm 0.4$. The error bars on these quantities are estimates of systematic uncertainties due to corrections to scaling.

The value of $\nu$ determined previously $\theta$ for a bounded distribution random fields, $\nu=3.0 \pm 0.5$, is consistent with our result. However, this value was determined from the fingerwidth and is not reliable. Future work is needed to determine whether bounded and unbounded distributions are in the same universality class.

Perković et a 9 , 0 have determined the critical behavior for the 3D Gaussian RFIM using a growth algorithm that appears to be in a different universality class. They analyzed the integrated avalanche size distribution occurring in one branch of a hysteresis loop ( $H$ increasing from $-\infty$ to $\infty)$. From the divergence as $\Delta$ decreased to $\Delta_{c}$ they found numerical values for the critical disorder, $\Delta_{c}=2.16 \pm 0.03$, and correlation length exponent, $\nu=1.43 \pm 0.18$.

The discrepancy between our results and those of Perković et al. seems to result from a crucial difference in our growth algorithms. They allowed any spinflip that lowered the energy, while we only allowed spins on the interface to flip. The exchange coupling between neighbors dominates in the low disorder limit, and spins are very unlikely to flip unless they are on the interface. Nowak et al.15. 16 found that the difference between the two algorithms was negligible in simulations of low disorder growth with uniform disorder. The two algorithms should also yield the same percolating cluster in the high disorder limit, where interactions between neighbors become irrelevant. The problem maps onto ordinary percolation, and the order in which spins are flipped becomes irrelevant. Near $\Delta_{c}$ these arguments break down, and the algorithms may give different results. At intermediate disorder, the exchange coupling is weak enough to allow clusters to flip ahead of the interface, and correlations are important enough that these flipped clusters can aid the advance of the approaching interface. One expects that both $H_{c}$ and $\Delta_{c}$ will be lowered by the advance clusters in Perković et al.'s model, and this is consistent with the numerical results.

\section{ACKNOWLEDGMENTS}

This work was partially supported by CNPq, CAPES, FAPERJ and FUJB (Brazil), by National Science Foundation Grant DMR 9634131, and by Intel Corporation through the donation of workstations that were used for our simulations. We thank G. Magnusson for assistance in implementing the growth algorithm, and R. Paredo and C. S. Nolle for useful conversations.

${ }^{1}$ F. Family and T. Vicsek, "Dynamics of Fractal Surfaces," (World Scientific, Singapore, 1991).

2 A.-L. Baraási and H. E. Stanley, "Fractal Concepts in Surface Growth," (Cambridge, Cambridge, 1995).

${ }^{3}$ M. Cieplak and M. O. Robins, Phys. Rev. Lett. 60, 2042 (1988); Phys. Rev. B 41, 11508 (1990).

${ }^{4}$ N. Martys, M. Cieplak, and M. O. Robbins, Phys. Rev. Lett. 66, 1058 (1991); N. Martys, M. O. Robbins, and M. Cieplak, Phys. Rev. B 44, 12294 (1991).

${ }^{5}$ H. Ji and M. O. Robbins, Phys. Rev. B 44, 2538 (1991).

${ }^{6}$ H. Ji and M. O. Robbins, Phys. Rev. B 46, 14519 (1992).

${ }^{7}$ B. Koiller, H. Ji, and M. O. Robbins, Phys. Rev. B46, 5258 (1992).

${ }^{8}$ B. Koiller, H. Ji, and M. O. Robbins, Phys. Rev. B45, 7762 (1992).

${ }^{9}$ O. Perković, K. A. Dahmen, and J. P. Sethna, Phys. Rev. Lett. 75, 4528 (1995).

${ }^{10}$ O. Perković, K. A. Dahmen, and J. P. Sethna, Phys. Rev. B59, 6106 (1999).

11 J. P. Stokes, D. A. Weitz, J. P. Gollub, A. Dougherty, M. O. Robbins, P. M. Chaikin, and H. M. Lindsay, Phys. Rev. Lett. 57, 1718 (1986).

12 T. Emig and T. Nattermann, Phys. Rev. Lett. 81, 1469 (1998).

13 N. Martys, "Critical Phenomena in Fluid Invasion of Porous Media," Ph.D. Thesis, Johns Hopkins University, 1990.

${ }^{14}$ L. A. N. Amaral, A.-L. Barabási, and H. E. Stanley, Phys. Rev. Lett. 73, 62 (1994).

${ }^{15}$ U. Nowak and K. D. Usadel, Europhys. Lett. 44, 634 (1998).

${ }^{16}$ L. Roters, A. Hucht, S. Lubeck, U. Nowak, and K. D. Usadel, Phys. Rev. E 60, 5202 (1999).

17 C. S. Nolle, B. Koiller, N. Martys, and M. O. Robbins, Phys. Rev. Lett. 71, 2074 (1993); Physica A 205, 342 (1994).

18 C. S. Nolle, "The Effects of Quenched Disorder on Moving Interfaces," PhD thesis, Johns Hopkins University, 1996.

19 J. Adler, Y. Meir, A. Aharony and A. B. Harris, Phys. Rev. B41, 9183 (1990).

${ }^{20}$ Note that the value of $P_{c}$ depends on the ratio betwegn the height and width of the cell, but $H_{c}$ does not (Ref.18).

${ }^{21}$ G. Grinstein and S. Ma, Phys. Rev. B28, 2588 (1983) determine $\alpha=(5-d) / 3$ in $d$-dimensions.

${ }^{22}$ O. Narayan and D. S. Fisher, Phys. Rev. B48, 7030 (1993).

23 T. Nattermann, S. Stepanow, L. H. Tang and H. Leschorn, J. Phys. II, 2, 1483 (1992). 